Graphene based integrated tandem supercapacitors fabricated directly on separators

Wei Chen, Chuan Xia, H.N. Alshareef

PII: S2211-2855(15)00144-5

DOI: $\quad$ http://dx.doi.org/10.1016/j.nanoen.2015.03.040

Reference: NANOEN789

To appear in: Nano Energy

Received date: 5 March 2015

Revised date: 28 March 2015

Accepted date: 28 March 2015

Cite this article as: Wei Chen, Chuan Xia, H.N. Alshareef, Graphene based integrated tandem supercapacitors fabricated directly on separators, Nano Energy, http://dx.doi.org/10.1016/j.nanoen.2015.03.040

This is a PDF file of an unedited manuscript that has been accepted for publication. As a service to our customers we are providing this early version of the manuscript. The manuscript will undergo copyediting, typesetting, and review of the resulting galley proof before it is published in its final citable form. Please note that during the production process errors may be discovered which could affect the content, and all legal disclaimers that apply to the journal pertain. 


\title{
Graphene based integrated tandem supercapacitors fabricated directly on separators
}

\author{
Wei Chen ${ }^{+}$, Chuan Xia ${ }^{+}$, H. N. Alshareef*
}

Materials Science and Engineering, King Abdullah University of Science and Technology (KAUST), Thuwal 23955-6900, Saudi Arabia

*E-mail: husam.alshareef@kaust.edu.sa

${ }^{+}$Authors contributed equally to this work

Keywords: graphene; integrated device; tandem supercapacitors; separator; high volumetric energy density

\begin{abstract}
It is of great importance to fabricate integrated supercapacitors with extended operation voltages as high energy density storage devices. In this work, we develop a novel direct electrode deposition on separator (DEDS) process to fabricate graphene based integrated tandem supercapacitors for the first time. The DEDS process generates compact graphene-polyaniline electrodes directly on the separators to form integrated supercapacitors. The integrated graphenepolyaniline tandem supercapacitors demonstrate ultrahigh volumetric energy density of $52.5 \mathrm{Wh}$ $\mathrm{L}^{-1}$ at power density of $6037 \mathrm{~W} \mathrm{~L}^{-1}$ and excellent gravimetric energy density of $26.1 \mathrm{Wh} \mathrm{kg}^{-1}$ at power density of $3002 \mathrm{~W} \mathrm{~kg}^{-1}$ with outstanding electrochemical stability for over 10000 cycles. This study show great promises for the future development of integrated energy storage devices.
\end{abstract}




\section{Introduction}

The fast depletion of fossil fuel supplies and the ever-growing demand for energy in our society has made it essential that alternate renewable energy sources are developed.[1-4] By nature, many of the renewable energy sources are intermittent and it is therefore imperative that efficient energy storage technologies are developed. Rechargeable batteries have proven to be indispensable energy storage devices due to their high energy density. However, rechargeable batteries suffer from relatively low power density. On the other hand, supercapacitors are playing an increasingly important role in multiple energy storage applications due to their outstanding electrochemical performance, such as high power density, long shelf life and safe operation.[5-9] Supercapacitors can be used independently or coupled with batteries where their high power density can complement battery functions. Conventional porous carbon based supercapacitors (such as activated carbon in most commercial supercapacitors) store energy through ion adsorption on the surface of carbons by the formation of electrochemical double layers.[10-14] The capacitance of carbon supercapacitors is largely restrained by the limited ion adsorption capability of carbon, resulting in relatively low gravimetric energy density of 5 to $10 \mathrm{Wh} \mathrm{kg}^{-1}$ (or volumetric energy density of 5 to $\left.8 \mathrm{Wh} \mathrm{L}^{-1}\right)$. $[15,16]$ It has been recently recommended that the packing volumetric energy density (with unit of $\mathrm{Wh} \mathrm{L}^{-1}$ ) involving the entire supercapacitor components should be used as a practical parameter than the gravimetric one (with unit of Wh $\mathrm{kg}^{-1}$ ) to realistically evaluate supercapacitor devices.[17, 18] The volumetric energy density is a key performance metric in applications where the volume of the supercapacitor devices must be minimized, such as in consumer electronics and electrical vehicles.[19] Therefore judicious fabrication strategies of supercapacitors must be adopted to minimize cell volume and increase packing density. Aqueous supercapacitors have many intrinsic advantages over organic 
supercapacitors such as higher ionic conductivity, faster charge process, ease of fabrication and environmental benignity. However, their output voltages are mainly limited by the decomposition of the aqueous electrolytes.[20] Devices with multiple supercapacitors connected externally in series can achieve higher voltages, but this would lead to unavoidable energy losses, system resistances and packing volume increase.[21] It has so far been a great challenge to fabricate integrated supercapacitors with high output voltage and great volumetric energy density, although tremendous progress have been achieved recently on improving supercapacitor volumetric energy density. For example, Yan et al. prepared template-assisted graphene at low temperature and obtained high volumetric energy density of $27.2 \mathrm{Wh}^{-1}$ in aqueous electrolyte.[22] Han et al. introduced a scalable method to synthesis holey graphene as dense supercapacitor electrodes with volumetric energy density of $12 \mathrm{Wh} \mathrm{L}^{-1}$.[23] Yang et al. recently reported graphene supercapacitors with ultrahigh volumetric energy density of $60 \mathrm{Wh} \mathrm{L}^{-1}$ in ionic liquid electrolyte by creating a dense graphene film (with mass density of $1.25 \mathrm{~g} \mathrm{~cm}^{-3}$ ).[24] Microsupercapacitors fabricated by lithography present an excellent approach toward integrated devices with improved electrochemical performance.[25-29] However, lithography may be too costly an approach for large-scale fabrication of supercapacitors. It is therefore very important to develop a cost-effective process for the facile fabrication of tandem supercapacitors with high volumetric energy density.

In this work, we demonstrate a facile scalable process to fabricate graphene based integrated tandem supercapacitors with very high volumetric energy densities. The integrated supercapacitors were fabricated using a direct electrode deposition on separator process (henceforth referred to as DEDS process). It is worth noting that the vacuum filtration based electrode deposition approach has applied to prepare integrated electrodes for energy storage 
devices recently. For example, Weng et al. reported a graphene-cellulose paper supercapacitor by fabrication of graphene-cellulose papers as electrodes using vacuum filtration of the graphene solution.[30] Kang et al. prepared carbon nanotube coated nanocellulose papers as integrated electrodes by the vacuum filtration for all-solid-state flexible supercapacitors.[31] In the area of rechargeable batteries, it was recently reported by Zhou et al. that the capacity and cycle life of lithium-sulfur battery can be largely improved by designing an integrated structure of sulfur and graphene deposit on one side of separator, where the deposited graphene membrane plays as a conductive internal current collector.[32, 33] These electrode deposition methods provided good directions to fabricate integrated electrodes, however, the integrity of supercapacitors in a device level has not been explored. Different from the reported studies, in our fabrication process of integrated supercapacitors, compact graphene films were used to coat on both sides of a commercial separator follow the steps of vacuum filtration, ion exchange and capillary compression to form a single integrated supercapacitor cell. These single supercapacitor cells are then internally connected to make tandem supercapacitors (see Experimental Section for details). As shown in Figure 1a, the commercial separator has a porous structure, which functions as an excellent filtration membrane that serves two purposes: (1) it acts as a substrate on which graphene-based electrodes are deposited, and (2) it simultaneously employs as the separator in the resulting device. Thus, both the supercapacitor electrodes and the supercapacitor cell are created in a single step (Figure 1a). The "all-in-one" device possesses a unique structure that has not been reported before, in which two layers of the condensed graphene based films bind firmly to the porous separator, making the whole device highly compact (Figure 1a). Further, internal connection of multiple single graphene-on-separator cells gives rise to the fabrication of integrated tandem supercapacitors, as shown in Figure 1b. The assembled tandem supercapacitor 
consists of multiple cells that are laminated in series, forming a highly integrated device toward miniaturization of the supercapacitor by largely reduced device volume (Figure 1c). A digital photograph of the real tandem supercapacitor is shown in Figure S1.

\section{Experimental section}

\section{Preparation of graphene and polyaniline}

The well dispersed graphene solution was prepared by hydrothermal reduction of graphene oxide (produced by a modified Hummer's method) in basic media according to our previous work.[34] Polyaniline nanofibers were prepared by an oxidative template method as described elsewhere.[35]

\section{Fabrication of integrated supercapacitors on commercial separators}

The integrated graphene supercapacitors were fabricated by vacuum filtration of the graphene suspension on both sides of a commercial separator (Celgard 3501). The separator was served as a filtration membrane as well as the final separator as a part of the integrated supercapacitor. The filter was immediately disconnected upon the exhaustion of the residue dispersion on one side of the separator. The other side of the separator was also coated with a graphene film by the same filtration method. After carefully peeled off from the filter support, the integrated graphene-on-separator was transferred to $1 \mathrm{M} \mathrm{H}_{2} \mathrm{SO}_{4}$ electrolyte for the exchange of the interior water with electrolyte.[24] Subsequently, the integrated graphene-on-separator was placed between two Au foils and compressed by a pressure of $~ 100 \mathrm{MPa}$ with the help of clip binders to make dense graphene electrodes on the integrated supercapacitor. For the assembly of tandem cells, a layer of polyethylene (PE) foil attached on the edge of Au foil was placed tween two adjacent graphene-on-separator for the internal connection of the cells in series 
and preventing the fluid of electrolyte across the adjacent graphene-on-separator cells to avoid the shortage of the device.[36] In the case of integrated graphene-PAni supercapacitors, PAni nanofibers were firstly dispersed in the as-prepared graphene solution by sonication for $0.5 \mathrm{~h}$. The ratio of graphene-PAni composites can be controlled by the feeding amount of PAni and graphene in the suspension. Then the graphene-PAni suspension was vacuum filtered on both sides of separator by the similar method as the preparation of the integrated graphene-onseparator to produce graphene-PAni-on-separator for the tandem supercapacitors.

\section{Electrochemical measurements}

The electrochemical tests of the integrated supercapacitors were conducted at room temperature in a VMP3 electrochemical workstation (Bio-Logic) by the techniques of electrochemical impedance spectroscopy (EIS), cyclic voltammetry (CV) and galvanostatic charge-discharge (CD). $1 \mathrm{M} \mathrm{H}_{2} \mathrm{SO}_{4}$ was used as the electrolyte for all supercapacitor devices. The calculation of all parameters of the supercapacitors are displayed as following by taking 3-cell tandem supercapacitor as an example. The absolute capacitance (C in F) of the supercapacitor is obtained from the galvanostatic charge-discharge curves according to the following equations ${ }^{3}$ :

$$
C=\frac{I}{\frac{\Delta V}{\Delta t}}
$$

where $I$ is the constant current for charge-discharge and $\frac{\Delta V}{\Delta t}$ is slope of the discharge curve. Since the tandem supercapacitor is composed of three single cells connected in series, the absolute capacitance of each cell is $3 \mathrm{C}$. Thus, its cell capacitance (gravimetric, $\mathrm{F} \mathrm{g}^{-1}$; or volumetric, $\mathrm{F} \mathrm{cm}^{-3}$ ) based on the two electrodes is:

$$
C_{\text {cell }}=\frac{3 C}{2 x}
$$


where $x$ can be either the mass loading of a single electrode (materials loading on a single side of the separator) or the volume of a single electrode. Specific capacitance is the gravimetric capacitance with respect to a single electrode. Therefore, the total capacitance of the tandem supercapacitor is:

$$
\begin{gathered}
\frac{1}{C_{\text {total }}}=\frac{1}{C_{\text {cell }}}+\frac{1}{C_{\text {cell }}}+\frac{1}{C_{\text {cell }}} \\
C_{\text {total }}=\frac{C_{\text {cell }}}{3}
\end{gathered}
$$

Energy density:

$$
E=\frac{C_{t o t a l} * V^{2}}{2}
$$

Power density:

$$
P=\frac{E}{t}
$$

where $t$ is discharge time from CD curves.

\section{Materials characterization}

The morphology and microstructure were investigated by SEM (Nova Nano 630, FEI) and TEM (Titan 80-300 kV (ST) TEM, FEI). The Raman spectroscopy was conducted on a Hariba LabRAM HR spectrometer. X-ray diffraction was carried out by Bruker D8 Advance bulk power XRD. 


\section{Results and discussion}

The graphene nanosheets used as building blocks to fabricate the integrated supercapacitors exhibit typical two-dimensional ultrathin characteristics (Figure 2a).[37, 38] The well-dispersed graphene suspension obtained by reduction of graphene oxide in alkaline media is very stable ( $\mathrm{pH}$ of $\sim 10$, inset picture in Figure 2a), as has been observed in other studies.[34, 39] The PAni nanofibers used in this study show porous surface with a caterpillar-like feature that may facilitate the access of the electrolyte to the electrodes (Figure 2b).[35, 40] The PAni nanofibers have an average diameter of about $150 \mathrm{~nm}$. The freshly prepared PAni suspension was stable (inset picture in Figure 2b). However, due to its high concentration and the acidic environment ( $\mathrm{pH}$ of $\sim 4$ ), the PAni nanofibers in the suspension tend to aggregate and some precipitates were observed after sitting for $24 \mathrm{~h}$ (Figure S2a). Nevertheless, the mixture of graphene and PAni with the help of sonication results in stable graphene-PAni suspension (inset picture in Figure 2c), even after sitting for a long time (Figure S2a). This agrees well with the stable graphene-PAni suspension reported by Xu et al.[41] The PAni nanofibers are sandwiched between the graphene nanosheets and thus function as efficient spacers to avoid restacking of the graphene sheets (Figure 2c). As a result, we are able to make very stable graphene-PAni suspension in a large amount by this scalable method (Figure S2b). The PAni nanofiber was chosen as electrode material for the tandem graphene-PAni supercapacitors due to several attractive characteristics of PAni, such as good chemical and mechanical characteristics, high theoretical capacity, good electrical conductivity, and high flexibility. In addition, the graphenePAni suspensions used in our study were highly stable and allowed for easy preparation of graphene-PAni composite films on-separator cells. Furthermore, the PAni nanofibers proved to 
be effective spacers for preventing the restacking of graphene nanosheets, leading to porous but compact graphene-PAni films, as will be discussed later.

The separators used for the fabrication of compact tandem supercapacitors are commercial polypropylene membranes with thickness of $25 \mu \mathrm{m}$ and porosity of $55 \%$ (inset picture in Figure 2d). Looking into the surface of the separator, one can see that it exhibits irregular arrays of pores with narrow lateral pore sizes ranging between $50 \mathrm{~nm}$ and $100 \mathrm{~nm}$ (Figure 2d and Figure S2c), much smaller than the size of the graphene nanosheets (roughly between $500 \mathrm{~nm}$ and a few micrometers). These characteristics make the separator ideal for small ion penetration and excellent substrate for the deposition of graphene and graphene-PAni electrodes on it by vacuum filtration. The resulting graphene-PAni-on-separator cell (inset picture in Figure 2e and Figure S2d) shows two identical graphene-PAni electrodes attached closely on both sides of the separator. The integrated cell is highly flexible and portable owing to the inherent advantages of the separator. As shown in the cross sectional image of the graphenePAni-on-separator cell (Figure 2e), the graphene-PAni compact nanocomposite layer was strongly bound to the surface of the separator. This result is due to the fact that ultra-filtration of the suspension and the ion exchange process generate condensed graphene-PAni film that bonds firmly with the separator. Furthermore, physical compression of the stacks improves the physical integrity and compactness of the device. As a result, the mass density of our graphenePAni film is as high as $2 \mathrm{~g} \mathrm{~cm}^{-3}$, higher than that of the compact graphene film (up to $1.49 \mathrm{~g} \mathrm{~cm}^{-3}$ ) and the graphene-PAni film $\left(1.53 \mathrm{~g} \mathrm{~cm}^{-3}\right)$ reported in the good studies by other groups.[24, 41]

The graphene-PAni was further characterized by X-ray diffraction (XRD) and Raman spectroscopy. The XRD of graphene shows a sharp peak of (002) around $26^{\circ}$ and the PAni exhibits three broad characteristic peaks centered around $16^{\circ}, 20.5^{\circ}$ and $25.5^{\circ}$ (Figure S3).[42] 
The composite of graphene-PAni as a whole shows the combined features of graphene and PAni, indicating the homogeneous mixture of the composites.[43] Moreover, the Raman spectra of graphene-PAni show the characteristic peaks from both graphene (D and $G$ bands) and PAni (centered around $1168 \mathrm{~cm}^{-1}, 1333 \mathrm{~cm}^{-1}$ and $1458 \mathrm{~cm}^{-1}$ ), which agrees well with the reported graphene-PAni composites.[44]

The electrochemical performance of integrated graphene tandem supercapacitors is demonstrated in Figure 3 and Figure S4. The single graphene-on-separator cell (henceforth referred to as 1-cell graphene supercapacitor) shows excellent electrochemical performance with nearly ideal double-layer capacitor behaviors: rectangular cyclic voltammetry curves at different scan rates (Figure $\mathrm{S} 4 \mathrm{a}$, from 50 to $500 \mathrm{mV} \mathrm{s}^{-1}$ ) and symmetric charge and discharge profiles over various current densities (Figure S4b, from 10 to $100 \mathrm{~A} \mathrm{~g}^{-1}$ ). However, the working voltage of the 1-cell graphene supercapacitor is only $1 \mathrm{~V}$. It is worth noting that the energy density of a supercapacitor is proportional to the square of output voltage $\left(\mathrm{E}=0.5 \mathrm{CV}^{2}\right)$. Thus, it is very crucial to extend the working voltage while keeping good cyclability. We therefore developed an approach by internal connection of the integrated graphene-on-separator cells to make graphene tandem supercapacitors with higher output voltages for higher energy density. The 2-cell graphene supercapacitor, made by internal connection of two graphene-on-separator cells in series, shows working voltage of $2 \mathrm{~V}$ and good electrochemical energy storage performance (Figure S4c and S4d). The voltage of the aqueous integrated graphene tandem supercapacitors was further extended to $3 \mathrm{~V}$ as shown in the 3-cell graphene supercapacitor (Figure S4e and S4f). As summarized in Figure 3a and 3b, compared to the 1-cell graphene supercapacitor, the 2 and 3cell tandem supercapacitors show similar good electrochemical performance at the same scan rate of $100 \mathrm{mV} \mathrm{s}^{-1}$ and current density of $20 \mathrm{~A} \mathrm{~g} \mathrm{~g}^{-1}$, and the voltage windows were steadily 
enhanced to higher levels. The impedance of the graphene tandem supercapacitors behavior is as good as the single cell one, but their charge transfer resistances were increased due to the longer charge transfer distance caused by the internal cell connection. The electrochemical cyclability of the graphene tandem supercapacitor (Figure 3d) shows outstanding stability (97.2\% capacity retention after 10000 cycles) as good as the single cell one (99.3\% capacity retention after 10000 cycles), indicating the successful fabrication of the integrated tandem supercapacitors for high energy density devices. It is noting that although there is a capacitance fluctuation in the 1-cell graphene device, the fluctuation is within only $4 \%$, which is not a large fluctuation and has negligible influence on the long-term stability of the device (Figure 3d). The slight capacitance fluctuation of the 1-cell graphene device is probably due to the gradual infiltration of the electrolyte into the compact graphene film until it being fully wetted.

The graphene-PAni tandem supercapacitors were fabricated by the same strategy as that of the graphene tandem supercapacitors. A series ratios of graphene to PAni were explored to find out the optimized composition of graphene-PAni composites as electrode. As shown in Figure S5, as the ratio of PAni to graphene increases in the composite, the specific capacitance of the graphene-PAni supercapacitor increases. However, when the amount of PAni reached a critical percentage of about $82.3 \%$, the graphene-PAni suspension became unstable and the resulting films were hard to form by vacuum filtration. In order to maximize the capacity while maintaining good mechanical property, the ratio of the graphene-PAni composite was determined to be $82.3 \%$ of PAni and we therefore focused on studying this composition to fabricate the tandem supercapacitors.

Figure 4 and S6 show the electrochemical performance of the graphene-PAni tandem supercapacitors. These devices exhibit excellent electrochemical properties even at high scan rate 
of $500 \mathrm{mV} \mathrm{s}^{-1}$ and high current density of $100 \mathrm{~A} \mathrm{~g}^{-1}$, revealing their good capability for fast charging and discharging (Figure S6). Different from the graphene tandem supercapacitors, the graphene-PAni cells showed two pairs of redox peaks during the charge and discharge processes, corresponding to the Faradaic transformation of the emeraldine-pernigraniline form of polyaniline and indicating the impact of PAni in the composite of graphene-PAni (Figure S6).[44] The redox peak positions shifted to higher potentials when the voltage of the integrated graphene-PAni tandem supercapacitors doubled or tripled; however, the overall behaviors of the cyclic voltammetry curves were similar to the single graphene-PAni-on-separator cell (Figure 4a). Similar to the graphene-only tandem supercapacitors, the graphene-PAni tandem supercapacitors exhibit almost equal charge and discharge times at the same current density of 20 $\mathrm{A} \mathrm{g}^{-1}$ as of the single graphene-PAni-on-separator cell, indicating that the loss of capacitance is negligible when the single cell was scaled up for the tandem supercapacitors (Figure $4 \mathrm{~b}$ ). The contribution of PAni in the graphene-PAni supercapacitors became distinct when comparing the graphene-PAni supercapacitors with the graphene ones. Taking the 3-cell tandem supercapacitor as an example, the integrated area of the cyclic voltammetry curve (Figure S7a) and the duration of the charge-discharge curve (Figure S7b) of the graphene-PAni supercapacitor remained much larger than that of the graphene supercapacitor. As a result, the 3-cell graphene-PAni supercapacitor shows much higher specific capacitance (between 391 and $335 \mathrm{~F} \mathrm{~g}^{-1}$ ) than the graphene supercapacitor (between 192 and $161 \mathrm{~F} \mathrm{~g}^{-1}$ ) under different current densities (from 5 to $100 \mathrm{~A} \mathrm{~g}^{-1}$ ) as shown in Figure 4c. Meanwhile, the rate capability of the integrated 3-cell graphene-PAni tandem supercapacitor (85.6\%) is better than that of the graphene one (83.8\%), confirming the excellent electrochemical performance of the graphene-PAni tandem supercapacitors. In terms of the volumetric capacitance (based on the volume of active materials), 
the graphene-PAni supercapacitors show superior values than that of the graphene cells (Figure $4 \mathrm{c}$ and Figure S8). At low current density of $5 \mathrm{~A} \mathrm{~g}^{-1}$, the 1-cell graphene-PAni supercapacitor exhibits very high volumetric capacitance of $199 \mathrm{~F} \mathrm{~cm}^{-3}$ with film density of $2 \mathrm{~g} \mathrm{~cm}^{-3}$ (Figure 4d), much higher than the 1-cell graphene supercapacitor with volumetric capacitance of $32.5 \mathrm{~F} \mathrm{~cm}^{-3}$ and film density of $0.53 \mathrm{~g} \mathrm{~cm}^{-3}$ (Figure S8). For the 3-cell tandem supercapacitors, the graphenePAni supercapacitor shows also higher volumetric capacitance of $65.6 \mathrm{~F} \mathrm{~cm}^{-3}$ than the graphene supercapacitor $\left(8.6 \mathrm{~F} \mathrm{~cm}^{-3}\right)$. The high gravimetric and volumetric capacitances of our integrated graphene-PAni supercapacitors are among some of the best values reported so far from different materials.[24, 45-49] Since the actual device volume is a more practical parameter to evaluate the energy storage devices, we defined a so-called device volumetric capacitance by considering the volume of all components in the supercapacitors. Figure $4 \mathrm{~d}$ shows that the device volumetric capacitance are $21.5 \mathrm{~F} \mathrm{~cm}^{-3}$ for 1-cell and $7.1 \mathrm{~F} \mathrm{~cm}^{-3}$ for 3-cell graphene-PAni supercapacitors, respectively; while such values are only $2.4 \mathrm{~F} \mathrm{~cm}^{-3}$ for 1-cell and $0.6 \mathrm{~F} \mathrm{~cm}^{-3}$ for 3-cell graphene supercapacitors, respectively (Figure S8).

Energy and power densities (both gravimetric and volumetric) are important parameters in the supercapacitor energy storage applications. As shown in Figure 4e, our integrated 3-cell graphene-PAni tandem supercapacitors show very high gravimetric energy density of $26.1 \mathrm{Wh}$ $\mathrm{kg}^{-1}$ at power density of $3002 \mathrm{~W} \mathrm{~kg}^{-1}$, superior to that of 1-cell graphene-PAni supercapacitor with energy density of $8.8 \mathrm{Wh} \mathrm{kg}^{-1}$ at power density of $1002 \mathrm{~W} \mathrm{~kg}^{-1}$, or that of 3-cell graphene tandem supercapacitors with energy density of $20 \mathrm{Wh} \mathrm{kg}^{-1}$ at power density of $4448 \mathrm{~W} \mathrm{~kg}^{-1}$. Correspondingly, the integrated 3-cell graphene-PAni tandem supercapacitors exhibit superb volumetric energy density of $52.5 \mathrm{Wh} \mathrm{L}^{-1}$ at power density of $6037 \mathrm{~W} \mathrm{~L}^{-1}$, much higher than that of the 1-cell graphene-PAni supercapacitor with energy density of $17.7 \mathrm{Wh} \mathrm{L}^{-1}$ at power density 
of $2015 \mathrm{~W} \mathrm{~L}^{-1}$, and the 3-cell graphene tandem supercapacitors with energy density of $10.7 \mathrm{Wh}$ $\mathrm{L}^{-1}$ at power density of $2386 \mathrm{~W} \mathrm{~L}^{-1}$ (Figure $4 \mathrm{f}$ ). These volumetric energy and power densities are comparable to the best performance reported so far.[24] Meanwhile, the device volumetric energy and power densities of our integrated 3-cell graphene-PAni tandem supercapacitors are still 5.7 $\mathrm{Wh} \mathrm{L}^{-1}$ and $651 \mathrm{~W} \mathrm{~L}^{-1}$ respectively (Figure S9), still much better than that of the 1-cell graphene-PAni supercapacitor (with device volumetric energy density of $1.9 \mathrm{Wh} \mathrm{L}^{-1}$ at power density of $217 \mathrm{~W} \mathrm{~L}^{-1}$ ), and the 3-cell graphene tandem supercapacitors (with device volumetric energy density of $0.8 \mathrm{Wh} \mathrm{L}^{-1}$ at power density of $176 \mathrm{~W} \mathrm{~L}^{-1}$ ). The integrated 3-cell graphenePAni tandem supercapacitors showed good cycling stability, retaining $92.5 \%$ of the initial capacity after 10000 cycles (Figure S10). It is noting that the device energy and power densities of the integrated tandem supercapacitor can be further increased by utilization of thinner separator or thicker active material films. To show the real energy storage application, our graphene-PAni tandem supercapacitor is capable of lighting a white LED when it is quickly charged within a few seconds, as shown in Figure S11.

\section{Conclusions}

In conclusion, high-performance graphene based tandem supercapacitors fabricated directly on separators were demonstrated for the first time. A facile and effective approach was developed to integrate porous graphene-PAni films (with mass density of $2 \mathrm{~g} \mathrm{~cm}^{-3}$ ) on the separator by a DEDS process involving vacuum filtration, ion exchange and capillary compression. The integrated graphene-PAni tandem supercapacitors showed very high specific capacitance of $391 \mathrm{~F} \mathrm{~g}^{-1}$ and volumetric capacitance of $199 \mathrm{~F} \mathrm{~cm}^{-3}$. Meanwhile, they delivered ultrahigh volumetric energy density of $52.5 \mathrm{Wh} \mathrm{L}^{-1}$ at power density of $6037 \mathrm{~W} \mathrm{~L}^{-1}$ and excellent 
gravimetric energy density of $26.1 \mathrm{Wh} \mathrm{kg}^{-1}$ at power density of $3002 \mathrm{~W} \mathrm{~kg}^{-1}$. Our study provides a general strategy towards future integrated energy storage devices.

\section{Acknowledgments}

Research reported in this publication has been supported by King Abdullah University of Science and Technology (KAUST). The authors wish to thank Dr. Rakhi RaghavanBaby and Dr. Narendra Kurra from the Functional Nanomaterials and Devices Group at KAUST for several useful discussions.

\section{Supporting Information}

Supplementary data associated with this article can be found in the online version at http://dx.doi.org.

\section{References}

[1] B. Dunn, H. Kamath, J. M. Tarascon, Science 334 (2011) 928-935.

[2] Z. Yang, J. Zhang, M. C. W. Kintner-Meyer, X. Lu, D. Choi, J. P. Lemmon, J. Liu, Chem. Rev. 111 (2011) 3577-3613.

[3] G. Wang, L. Zhang, J. Zhang, Chem. Soc. Rev. 41 (2012) 797-828.

[4] C. Liu, F. Li, L.-P. Ma, H.-M. Cheng, Adv. Mater. 22 (2010) E28-E62.

[5] P. Simon, Y. Gogotsi, Nat. Mater. 7 (2008) 845-854.

[6] M. Winter, R. J. Brodd, Chem. Rev. 104 (2004) 4245-4269.

[7] R. Kotz, M. Carlen, Electrochim. Acta 45 (2000) 2483-2498. 
[8] J. M. Tarascon, M. Armand, Nature 414 (2001) 359-367.

[9] A. S. Arico, P. Bruce, B. Scrosati, J. M. Tarascon, W. Van Schalkwijk, Nat. Mater. 4 (2005) 366-377.

[10] L. L. Zhang, X. S. Zhao, Chem. Soc. Rev. 38 (2009) 2520-2531.

[11] P. J. Hall, M. Mirzaeian, S. I. Fletcher, F. B. Sillars, A. J. R. Rennie, G. O. Shitta-Bey, G. Wilson, A. Cruden, R. Carter, Energy Environ. Sci. 3 (2010) 1238-1251.

[12] A. G. Pandolfo, A. F. Hollenkamp, J. Power Sources 157 (2006) 11-27.

[13] G. Yu, X. Xie, L. Pan, Z. Bao, Y. Cui, Nano Energy 2 (2013) 213-234.

[14] F. Bonaccorso, L. Colombo, G. Yu, M. Stoller, V. Tozzini, A. C. Ferrari, R. S. Ruoff, V. Pellegrini, Science 347 (2015) 1246501.

[15] A. Burke, Electrochim. Acta 53 (2007) 1083-1091.

[16] E. Frackowiak, PCCP 9 (2007) 1774-1785.

[17] P. Simon, Y. Gogotsi, B. Dunn, Science 343 (2014) 1210-1211.

[18] Y. Gogotsi, P. Simon, Science 334 (2011) 917-918.

[19] M. Armand, J. M. Tarascon, Nature 451 (2008) 652-657.

[20] X. H. Zhou, C. Peng, G. Z. Chen, AlChE J. 58 (2012) 974-983.

[21] Y. Gogotsi, Nature 509 (2014) 568-570.

[22] J. Yan, Q. Wang, T. Wei, L. Jiang, M. Zhang, X. Jing, Z. Fan, ACS Nano 8 (2014) 47204729.

[23] X. Han, M. R. Funk, F. Shen, Y.-C. Chen, Y. Li, C. J. Campbell, J. Dai, X. Yang, J.-W.

Kim, Y. Liao, J. W. Connell, V. Barone, Z. Chen, Y. Lin, L. Hu, ACS Nano 8 (2014) 8255-8265.

[24] X. W. Yang, C. Cheng, Y. F. Wang, L. Qiu, D. Li, Science 341 (2013) 534-537. 
[25] D. Pech, M. Brunet, H. Durou, P. Huang, V. Mochalin, Y. Gogotsi, P.-L. Taberna, P. Simon, Nat. Nanotech. 5 (2010) 651-654.

[26] C. Meng, J. Maeng, S. W. M. John, P. P. Irazoqui, Adv. Energy Mater. 4 (2014) 1301269.

[27] K. Wang, W. J. Zou, B. G. Quan, A. F. Yu, H. P. Wu, P. Jiang, Z. X. Wei, Adv. Energy Mater. 1 (2011) 1068-1072.

[28] G. P. Xiong, C. Z. Meng, R. G. Reifenberger, P. P. Irazoqui, T. S. Fisher, Electroanalysis 26 (2014) 30-51.

[29] N. Kurra, N. A. Alhebshi, H. N. Alshareef, Adv. Energy Mater. 5 (2015) 1401303.

[30] Z. Weng, Y. Su, D.-W. Wang, F. Li, J. Du, H.-M. Cheng, Adv. Energy Mater. 1 (2011) 917922.

[31] Y. J. Kang, S.-J. Chun, S.-S. Lee, B.-Y. Kim, J. H. Kim, H. Chung, S.-Y. Lee, W. Kim, ACS Nano 6 (2012) 6400-6406.

[32] G. Zhou, L. Li, D.-W. Wang, X.-y. Shan, S. Pei, F. Li, H.-M. Cheng, Adv. Mater. 27 (2015) 641-647.

[33] G. Zhou, S. Pei, L. Li, D.-W. Wang, S. Wang, K. Huang, L.-C. Yin, F. Li, H.-M. Cheng, Adv. Mater. 26 (2014) 625-631.

[34] Y. Bai, R. B. Rakhi, W. Chen, H. N. Alshareef, J. Power Sources 233 (2013) 313-319.

[35] Z. Liu, X. Zhang, S. Poyraz, S. P. Surwade, S. K. Manohar, J. Am. Chem. Soc. 132 (2010) 13158-13159.

[36] F. Zhang, Y. Lu, X. Yang, L. Zhang, T. Zhang, K. Leng, Y. Wu, Y. Huang, Y. Ma, Y. Chen, Small 10 (2014) 2285-2292.

[37] Y. Huang, J. Liang, Y. Chen, Small 8 (2012) 1805-1834. 
[38] Y. Wang, Z. Shi, Y. Huang, Y. Ma, C. Wang, M. Chen, Y. Chen, J. Phys. Chem. C 113 (2009) 13103-13107.

[39] D. Li, M. B. Muller, S. Gilje, R. B. Kaner, G. G. Wallace, Nat. Nanotech. 3 (2008) 101-105.

[40] W. Chen, C. Xia, R. B. Rakhi, H. N. Alshareef, J. Power Sources 267 (2014) 521-526.

[41] Q. Wu, Y. Xu, Z. Yao, A. Liu, G. Shi, ACS Nano 4 (2010) 1963-1970.

[42] L. J. Pan, G. H. Yu, D. Y. Zhai, H. R. Lee, W. T. Zhao, N. Liu, H. L. Wang, B. C. K. Tee, Y.

Shi, Y. Cui, Z. N. Bao, Proceedings of the National Academy of Sciences of the United States of America 109 (2012) 9287-9292.

[43] W. Chen, R. B. Rakhi, H. N. Alshareef, Nanoscale 5 (2013) 4134-4138.

[44] D.-W. Wang, F. Li, J. Zhao, W. Ren, Z.-G. Chen, J. Tan, Z.-S. Wu, I. Gentle, G. Q. Lu, H.-

M. Cheng, ACS Nano 3 (2009) 1745-1752.

[45] X. Yang, J. Zhu, L. Qiu, D. Li, Adv. Mater. 23 (2011) 2833-2838.

[46] M. F. El-Kady, R. B. Kaner, Nat. Commun. 4 (2013) 1475.

[47] Z. S. Wu, K. Parvez, X. Feng, K. Müllen, Nat. Commun. 4 (2013)

[48] J. Chmiola, G. Yushin, R. Dash, Y. Gogotsi, J. Power Sources 158 (2006) 765-772.

[49] P. Simon, Y. Gogotsi, Acc. Chem. Res. 46 (2013) 1094-1103. 
(a)

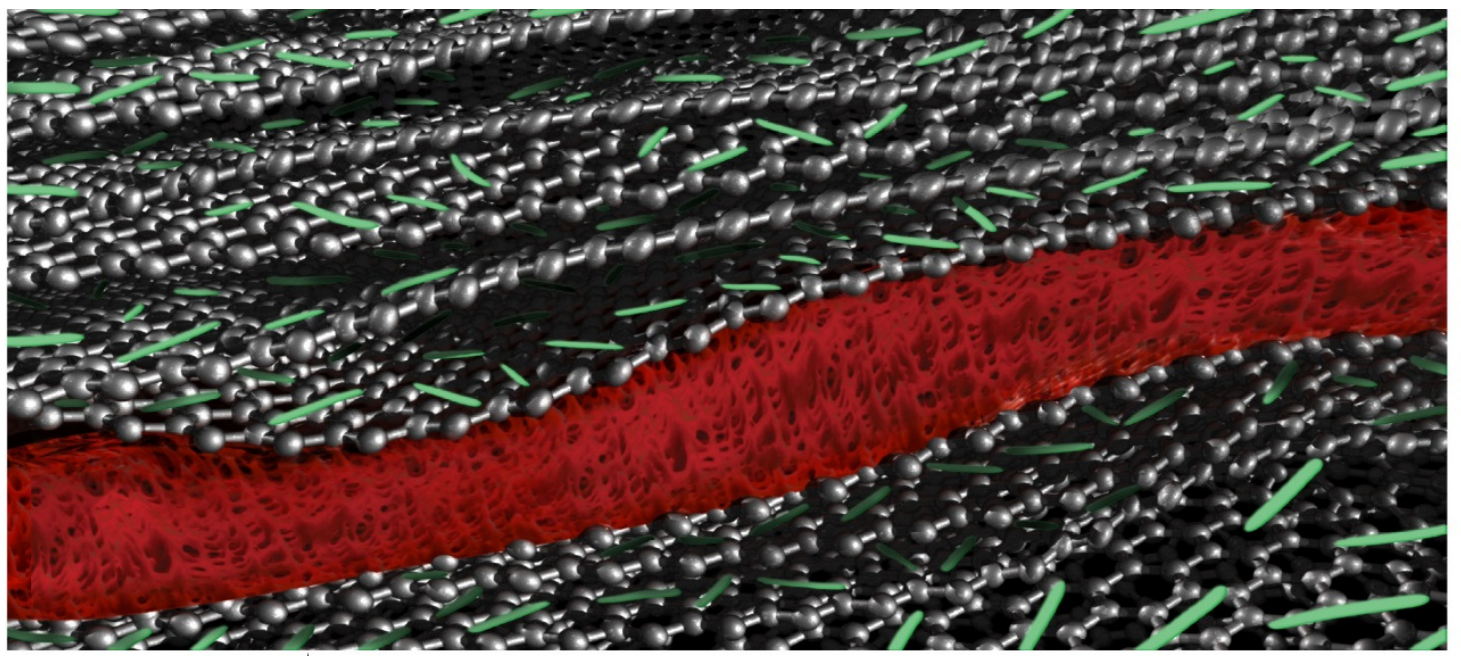

(b)

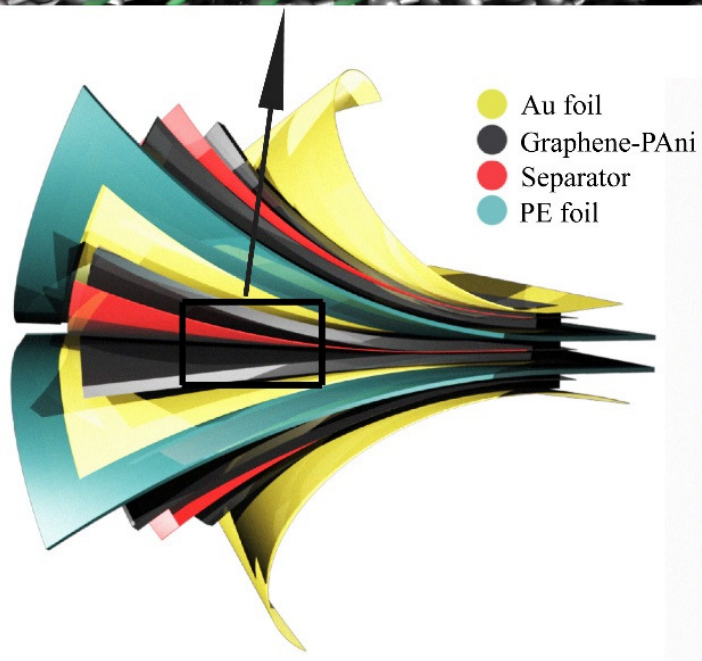

(c)

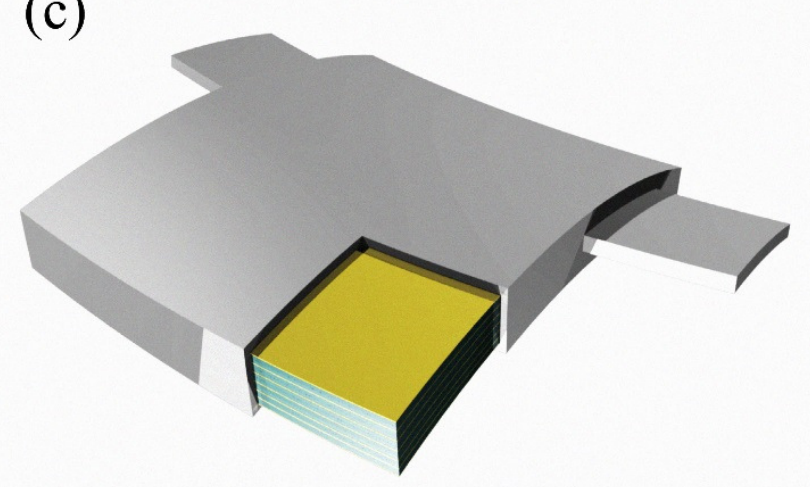

Figure 1. Schematic illustration of the fabrication of graphene based integrated tandem supercapacitors. (a) Integrated graphene-PAni-on-separator cell consisting of two compact graphene-PAni films deposited directly on both sides of a porous separator. The interior (b) and exterior (c) structures of the integrated graphene-PAni tandem supercapacitor. 

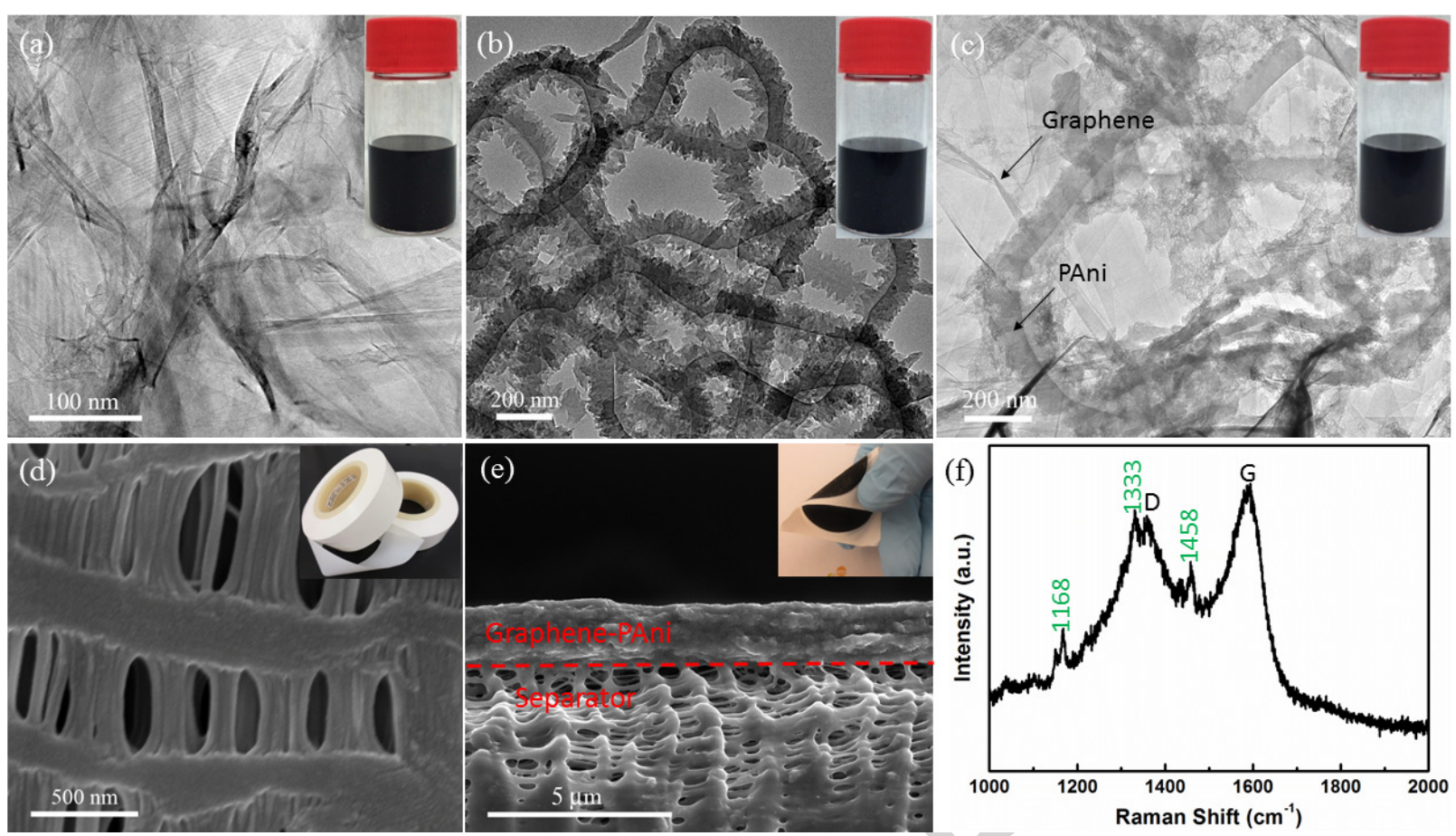

Figure 2. Characterization of the integrated graphene-PAni tandem supercapacitors. TEM images and digital photographs (inset) of (a) graphene nanosheets, (b) polyaniline nanofibers, and (c) graphene-polyaniline composites and their suspensions, respectively. SEM images and photographs (inset) of (d) the commercial separator and (e) the integrated graphene-PAni-onseparator cell. (f) Raman spectroscopy of the graphene-PAni composites. 

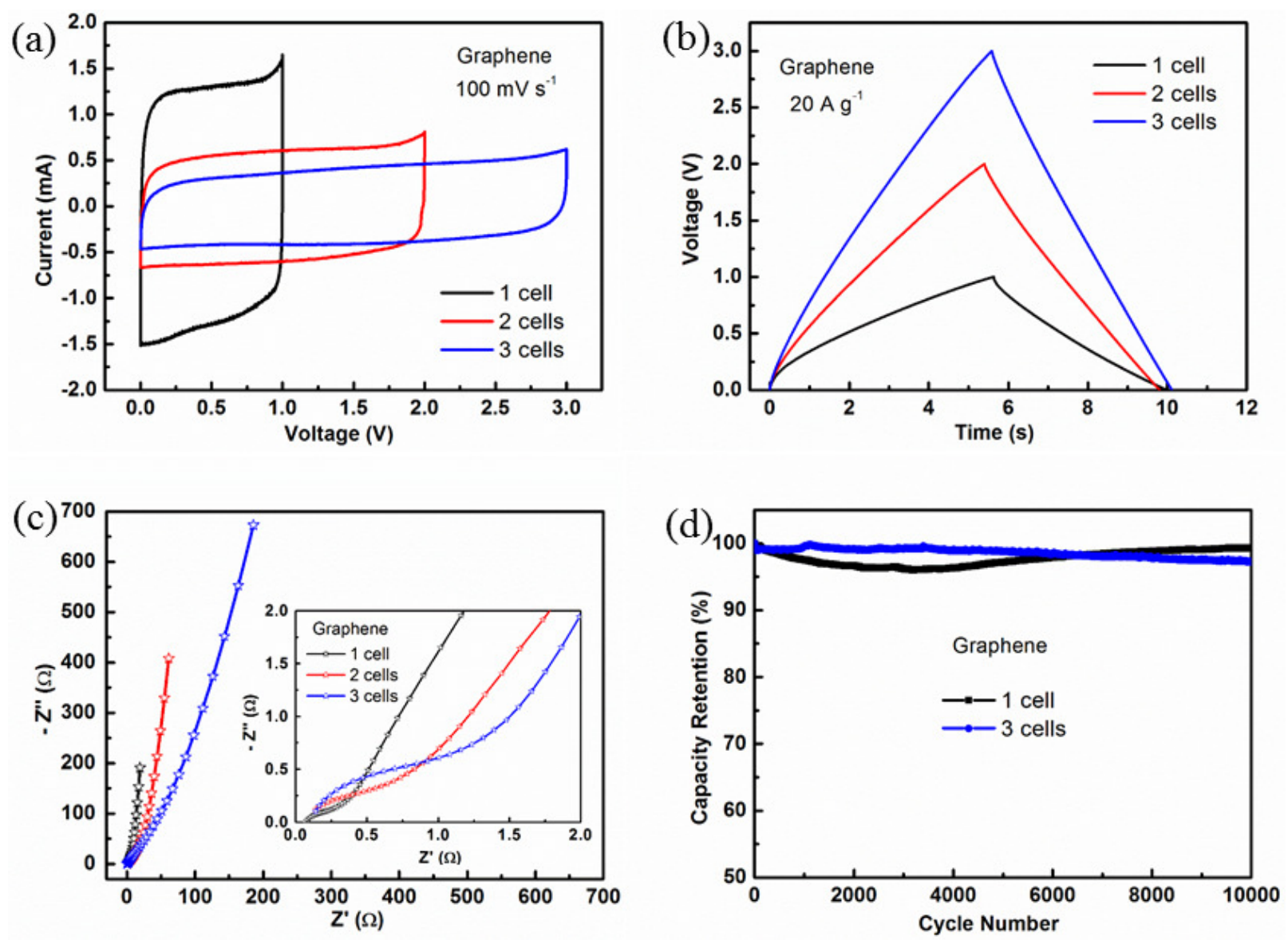

Figure 3. Electrochemical performance of the integrated graphene tandem supercapacitors. (a) Cyclic voltammetry at scan rate of $100 \mathrm{mV} \mathrm{s}^{-1}$; (b) galvanostatic charge-discharge curves at current density of $20 \mathrm{~A} \mathrm{~g}^{-1}$; (c) electrochemical impedance spectroscopy and (d) cycling stability behaviors. 

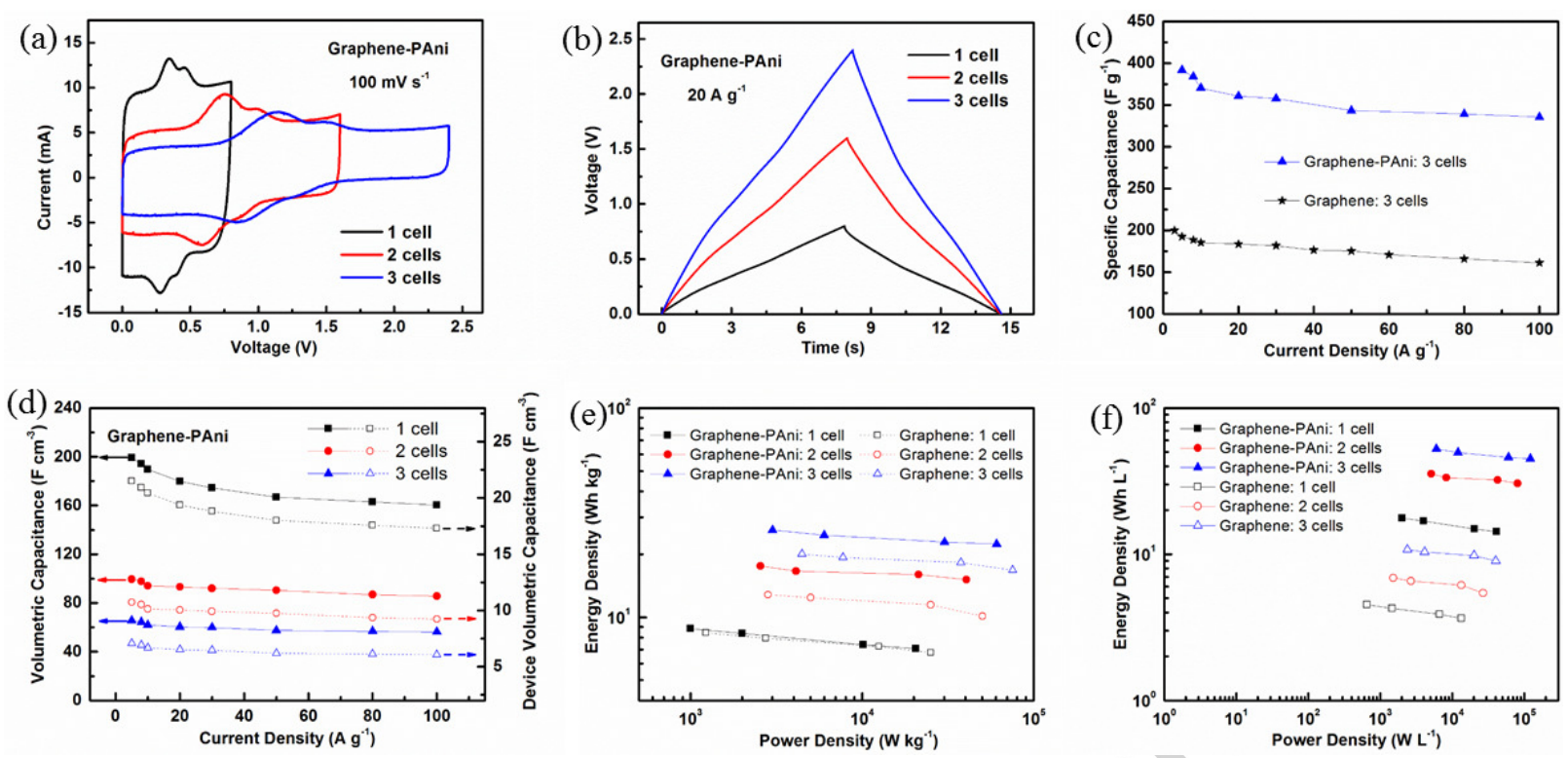

Figure 4. Electrochemical performance of the integrated graphene-PAni tandem supercapacitors.

(a) Cyclic voltammetry at scan rate of $100 \mathrm{mV} \mathrm{s}^{-1}$; (b) galvanostatic charge-discharge curves at current density of $20 \mathrm{~A} \mathrm{~g}^{-1}$; (c) Specific capacitance of the integrated 3-cell graphene and graphene-PAni tandem supercapacitors; (d) volumetric capacitance (normalized to active materials and the whole device) of the graphene-PAni tandem supercapacitors; (e) gravimetric energy and power density and (f) volumetric energy density for both integrated graphene and graphene-PAni tandem supercapacitors. 


\section{Graphical abstract}

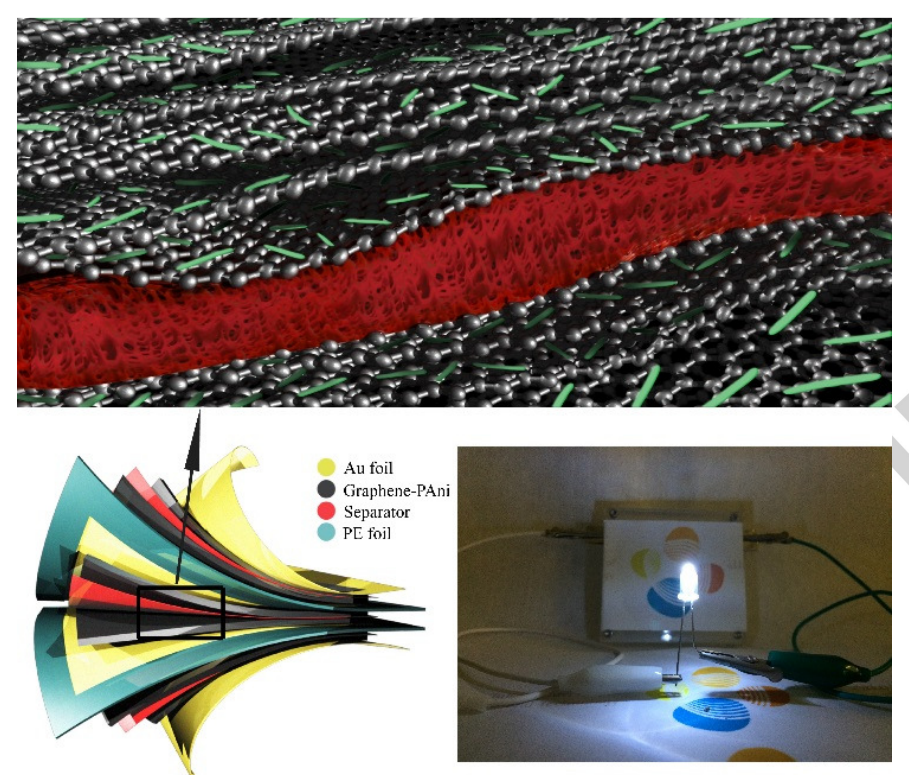

Graphene based integrated tandem supercapacitors are fabricated using direct electrode deposition on a separator (DEDS) process and demonstrated for the first time. The integrated graphene-polyaniline tandem supercapacitors show ultrahigh volumetric energy density of 52.5 $\mathrm{Wh} \mathrm{L}^{-1}$ at power density of $6037 \mathrm{~W} \mathrm{~L}^{-1}$ and excellent gravimetric energy density of $26.1 \mathrm{Wh} \mathrm{kg}^{-1}$ at power density of $3002 \mathrm{~W} \mathrm{~kg}^{-1}$ with outstanding stability for more than 10000 cycles. 


\section{Vitae}

\section{Wei Chen}

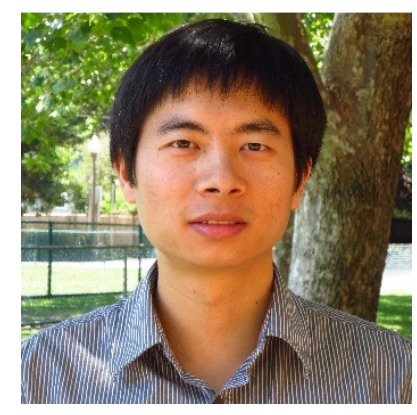

Wei Chen is currently a postdoctoral research fellow in the department of Materials Science and Engineering at Stanford University. Dr. Chen obtained his PhD from King Abdullah University of Science and Technology (KAUST) in 2013. His research interests include the development of nanostructured materials for rechargeable batteries, supercapacitors and electrocatalysts.

\section{Chuan Xia}

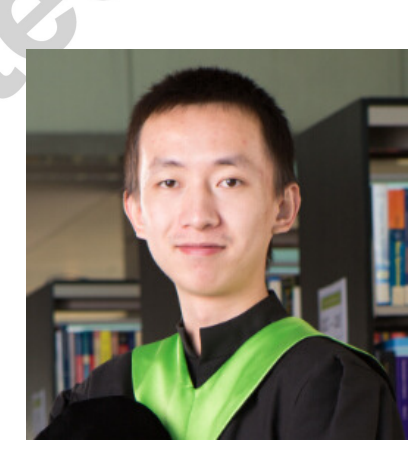

Chuan Xia is currently a PhD candidate of the Materials Science and Engineering program at KAUST. His research interests focus on the development of nanostructured chalcogenides and their applications. 


\section{Husam Alshareef}

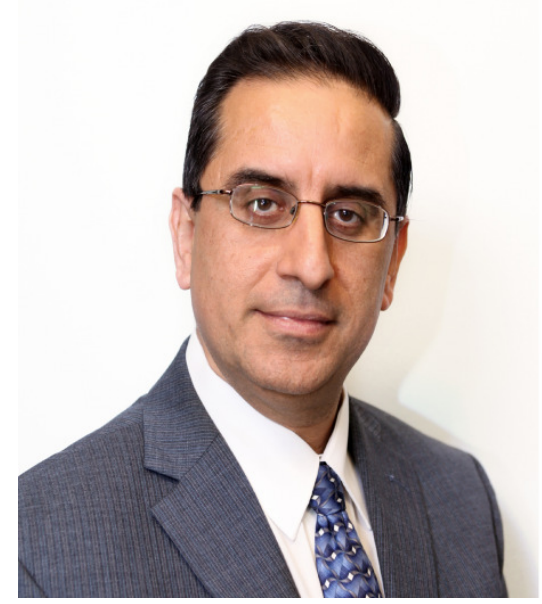

Husam Alshaeef is a professor and program chair in the Materials Science \& Engineering program at King Abdullah University of Science \& Technology (KAUST). He obtained his PhD at North Carolina State University, USA. His group is interested in the use of nanomaterials in electronics and energy applications. He is a frequent speaker at international conferences dealing with energy and electronics. 


\section{Highlights}

1. A novel direct electrode deposition on separator (DEDS) process is developed to fabricate graphene based integrated tandem supercapacitors for the first time.

2. The integrated graphene-polyaniline tandem supercapacitors show both very high volumetric and specific capacitances.

3. Ultrahigh volumetric energy and power densities can be achieved with outstanding long term cycling stability.

4. The strategy show great promises for the future development of integrated energy storage devices. 